\title{
Protease inhibitors show promise against $\mathrm{HCV}$
}

Data from trials presented at the 2008 American Association for the Study of Liver Diseases (AASLD) meeting have highlighted the potential of an emerging class of drugs for hepatitis $\mathrm{C}$ virus (HCV) that target the NS3/4 viral protease.

Current treatment of HCV consists of polyethylene glycol (PEG)ylated interferon agents combined with ribavirin. "However, in patients infected with HCV genotype 1 - the most common genotype worldwide treatment for 48 weeks with such combinations results in sustained virologic response (SVR) rates of only $40-50 \%$, emphasizing the need for more effective and better tolerated HCV therapies," says Stefan Zeuzem, Professor and Chief of Medicine at the J. W. Goethe University Hospital in Frankfurt, Germany.

The nonstructural NS3/4 serine protease, which processes the $\mathrm{HCV}$ polyprotein to generate mature viral proteins, has emerged as an attractive target for the development of new therapies (Nature Rev. Drug Discov. 6, 991-1000; 2007). Among the trials presented at the AASLD meeting was a study of telaprevir, a protease inhibitor that is currently in Phase III trials (TABLE 1). Patients infected with HCV genotype 1 who had failed to respond to standard therapy received either 24 weeks of telaprevir or 48 weeks of standard therapy. An interim analysis at 36 weeks showed that a combination of telaprevir, PEGylated interferon and ribavirin produced a $22 \%$ increase in SVR compared with controls. In another trial, combining boceprevir - which is also in late-stage development with PEGylated interferon and ribavirin for 48 weeks almost doubled the SVR in treatment-naive patients, compared with PEGylated interferon plus ribavirin alone.

Results so far with telaprevir and boceprevir suggest that triple combination regimens could lead to a new era in the treatment of most patients chronically infected with HCV genotype 1, says Zeuzem.

Nevertheless, there remain several important concerns, such as the ability of viral enzyme inhibitors in general to suppress all viral variants as well as to prevent the emergence of drug-resistant virus, says David Nelson, Professor of Medicine and Director of Hepatology and Liver Transplantation at the University of Florida, USA.

Finally, it is also noteworthy that a non-interferon-based Phase I safety trial has recently been initiated (TABLE 1). "It is not known whether HCV can be eradicated without an immunomodulatory drug to stimulate immune clearance from the liver. These interferon-free trials are anxiously awaited." says Nelson.

\footnotetext{
Table 1 | Selected protease inhibitors in HCV clinical trials

Drug (lead company) Update/status

Telaprevir (Vertex)*

Telaprevir triple therapy cohort (24-week therapy, genotype 1 treatment failure patients): $52 \%$ of patients achieved SVR ${ }_{12}$; standard therapy: $30 \%$ of patients achieved an SVR at week 36

Boceprevir (Schering-Plough)*

TMC-435 (Tibotec)*

MK-7009 (Merck)

PHX-1766 (Phenomix)

R7128/ITMN-191

(Roche)

ITMN-191 (InterMune)

VBY-376 (Virobay)

Boceprevir triple therapy cohort (48-week therapy, treatment-naive patients): $66 \%$ of patients achieved SVR ${ }_{12}$; boceprevir triple therapy cohort with 4-week standard therapy lead-in: $72 \%$ of patients achieved $\mathrm{SVR}_{12}$; standard therapy: $38 \%$ of patients achieved an SVR

Reduction in HCV RNA when used in triple therapy

Phase Il triple therapy in treatment-naive patients

Phase la initiation in healthy and HCV subjects

Phase la initiation of interferon-free NS5B polymerase inhibitor/NS3 protease inhibitor combination treatment in treatment-naive patients

Phase lb triple therapy in treatment-naive patients

Phase I safety trial in healthy subjects

*Phase II interim analysis presented at the 2008 American Association for the Study of Liver Diseases meeting. HCV, hepatitis C virus; standard therapy, polyethylene glycol (PEG)ylated interferon plus ribavirin for 48 weeks; SVR, sustained virologic response; SVR , SVR at 12 weeks post-treatment; triple therapy, investigational drug combined with PEGylated interferon plus ribavirin.
} 